\title{
On the Chiral Phase Transition in the Linear Sigma Model
}

\author{
Tran Huu Phat ${ }^{1)}$, Nguyen Tuan $\mathrm{Anh}^{2)}$ and Le Viet $\mathrm{Hoa}^{3)}$ \\ 1) Vietnam Atomic Energy Commission, \\ 59 Ly Thuong Kiet, Hanoi, Vietnam. \\ 2) Institute for Nuclear Science and Technique, \\ P.O.Box 5T-160, Hoang Quoc Viet, Hanoi, Vietnam \\ 3) Department of Physics, Hanoi University of Education II, Hanoi, Vietnam.
}

(July 2003)

\begin{abstract}
The Cornwall-Jackiw-Tomboulis (CJT) effective action for composite operators at finite temperature is used to investigate the chiral phase transition within the framework of the linear sigma model as the low-energy effective model of quantum chromodynamics (QCD). A new renormalization prescription for the CJT effective action in the Hartree-Fock (HF) approximation is proposed. A numerical study, which incorporates both thermal and quantum effect, shows that in this approximation the phase transition is of first order. However, taking into account the higher-loop diagrams contribution the order of phase transition is unchanged.

PACS numbers: $11.10 \mathrm{Wx}, 11.10 \mathrm{Gh}, 11.30 \mathrm{Rd}, 05.70 \mathrm{Fh}$.
\end{abstract}

\section{INTRODUCTION}

In recent years the study of the phase transitions has become the subject of intense investigation since it is very important from various aspects, ranging from cosmology to particle physics. According to the big bang model there is a series of phase transitions, including, of course, the chiral phase one, at the early stage of the universe evolution. The nature of the electroweak phase transition has called much attention due to the suggestion that the baryon asymmetry may be generated at the electroweak scale if the transition is of first order [1]. The restoration of chiral symmetry may lead to several interesting phenomena observed, for instance, in the dilepton mass spectrum [2] or in the formation of disoriented chiral condensates [3]. The quantum chromodynamics (QCD) is chirally symmetric for light quark sector and must be spontaneously broken due to the existence of pions, the Nambu-Goldstone (NG) bosons. The lattice QCD calculations indicate that the restoration of chiral symmetry occurs at temperatures of order $150 \mathrm{MeV}$ [4], which is expected to be probed in ultrarelativistic heavy-ion collision experiments planned at RHIC and LHC [5]. The field theoretical investigation of the symmetry restoration at finite temperatures was first carried out by Kirzhnits and Linde [6] and then was systematically developed by Weinberg [7], Dolan and Jackiw [8] as well as many others adopting the effective action as the most appropriate formalism for such studies. It was proved that the leading contributions at very high temperatures come from infinite series of certain class of multiloop diagrams in perturbation theory, which, in the $\lambda \phi^{4}$ theory, are the so-called daisy and superdaisy diagrams. Recently the effective action for composite operators, originally introduced by Cornwall, Jackiw and Tomboulis [9] at zero temperature, has been extended for finite temperatures in $\lambda \phi^{4}$ theory by Amelino-Camelia and Pi [10], who showed that one needs to resume only "double bubble" graphs instead of summing infinite set of daisy and superdaisy graphs using the tree level propagators. This is known as Hartree-Fock approximation 
[11]. Moreover, the CJT formalism at finite temperatures leads to reliable results [12, 13], among other approximate approachs.

Many works on chiral symmetry restoration at high temperatures have been accomplished [14-20] within the framework of the linear sigma model, which is considered to be the relevant model [21] for effective theory in low-energy phenomenology of QCD modelling the hadron dynamics associated to the chiral phase transition. However, there exists serious difficulty concerning the renormalization of the CJT effective action in the Hartree-Fock approximation. The point is that a consistent renormalization cannot be performed in the broken phase because all terms in the effective action are expressed not only by renormalized quantities but bare quantities remain in several terms [15]. In [16,19] one tried to regularize divergent integrals by means of several schemes instead of renormalizing the CJT effective action in Hartree-Fock approximation at finite temperatures.

In this work, dealing with the chiral phase transition at finite temperatures, we reexamine the linear sigma model since it is most suited for our purpose of describing the phase transition in a self-consistent approximation. We do not consider the general $O(N)$ linear sigma model at large-N limit because it significantly simplifies the counting of multiloop bubble diagrams, which is the most common source of inaccuracy.

We use the imaginary time formalism of Matsubara [22] and work in Euclidean spacetime. The Feynman rules are the same as those at zero temperature, except that

$$
\int \frac{d^{4} k}{(2 \pi)^{4}} f(k) \rightarrow \frac{1}{\beta} \sum_{n} \int \frac{d^{3} k}{(2 \pi)^{3}} f\left(\omega_{n}, \vec{k}\right) \equiv \int_{\beta} f(k),
$$

where $\omega_{n}=2 \pi n / \beta$.

This paper is organized as follows. Section II is devoted to calculating the CJT effective potential at finite temperatures in the Hartree-Fock approximation. Here a new renormalization prescription is proposed. A numerical computation is presented in Section III. The higher-loop diagram contribution in the vicinity of critical temperature is considered in Section IV. Finally conclusion and discussion are given in Section V.

\section{THE CJT EFFECTIVE POTENTIAL}

The lagrangian density of the $O(4)$ linear sigma model reads

$$
\mathcal{L}=\frac{1}{2}\left(\partial_{\mu} \phi^{\alpha}\right)^{2}+\frac{m^{2}}{2} \phi^{2}+\frac{\lambda}{24}\left(\phi^{2}\right)^{2}
$$

where $\phi^{2}=\phi^{\alpha} \phi^{\alpha} ; \phi^{i}=\pi^{i}, i=1,2,3 ; \phi^{4}=\sigma$.

The counterterms, which must be added to (II.1), are chosen as

$$
\Delta \mathcal{L}=\frac{\delta m^{2}}{2} \phi^{2}+\frac{\delta \lambda}{24}\left(\phi^{2}\right)^{2}+\delta \Omega
$$

in which the counterterm $\delta \Omega$ is introduced for vacuum energy. As a rule, all divergencies of the theory have to be absorbed by the counterterms (II.2).

By shifting the field as $\phi^{\alpha}(x) \rightarrow \phi^{\alpha}(x)+\phi_{0}^{\alpha}$, the tree-level propagators $D_{\alpha \beta}$ have the form 


$$
\begin{aligned}
D_{\alpha \beta}^{-1} & =\left.\frac{\delta^{2} I(\phi)}{\delta \phi^{\alpha}(x) \delta \phi^{\beta}(y)}\right|_{\phi=\phi_{0}} \\
& =\left[\partial_{\mu}^{2}+m^{2}+\delta m^{2}+\frac{\lambda+\delta \lambda}{6} \phi_{0}^{2}\right] \delta^{\alpha \beta} \delta^{4}(x-y)+\frac{\lambda+\delta \lambda}{3} \phi_{0}^{\alpha} \phi_{0}^{\beta} \delta^{4}(x-y) .
\end{aligned}
$$

Here $I(\phi)$ is the classical action

$$
I(\phi)=\int d^{4} x(\mathcal{L}+\Delta \mathcal{L})
$$

In momentum space, $D_{\alpha \beta}^{-1}$ is written as

$$
D_{\alpha \beta}^{-1}\left(k ; \phi_{0}\right)=\left[k^{2}+m^{2}+\delta m^{2}+\frac{\lambda+\delta \lambda}{6} \phi_{0}^{2}\right] \delta^{\alpha \beta}+\frac{\lambda+\delta \lambda}{3} \phi_{0}^{\alpha} \phi_{0}^{\beta} .
$$

The interaction action, describing the vertices of the shifted theory, is given by

$$
I_{\text {int }}\left(\phi, \phi_{0}\right)=\int d^{4} x\left[\frac{\lambda+\delta \lambda}{6} \phi^{2} \phi^{\alpha} \phi_{0}^{\alpha}+\frac{\lambda+\delta \lambda}{24}\left(\phi^{2}\right)^{2}\right] .
$$

Then, for constant $\phi_{0}$, we arrive at the CJT effective potential at finite temperatures

$$
V_{\beta}^{C J T}\left(\phi_{0}, G\right)=I\left(\phi_{0}\right)+\frac{1}{2} \int_{\beta} \operatorname{tr} \ln G^{-1}(k)+\frac{1}{2} \int_{\beta} \operatorname{tr}\left[D^{-1}\left(k ; \phi_{0}\right) G(k)\right]+V_{2}^{C J T}\left(\phi_{0}, G\right) .
$$

Here $G_{\alpha \beta}(k)$ is the full propagator of the theory and $V_{2}^{C J T}$ is the sum of all two and higher order loop two-particle irreducible vacuum graphs of the theory with vertices given by $I_{\text {int }}\left(\phi, \phi_{0}\right)$ and propagators set equal to $G_{\alpha \beta}(k)$. The graphs shown in Fig. 1 are under discussion. It is clear that, among them, only the two-loop graph, the "double bubble" one, of order $O(\lambda)$ includes contributions from daisy and superdaisy graphs of ordinary perturbation theory. It is known that truncating the series at $O(\lambda)$ is the Hartree-Fock approximation.
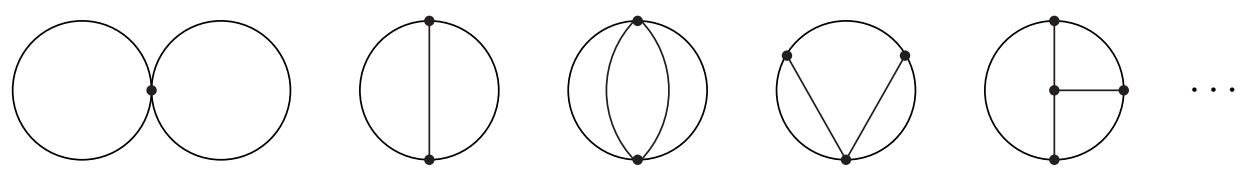

FIG. 1. Two-particle irreducible graphs contributing to $\Gamma_{2}(\phi, G)$ up to the three-loop level in a $\lambda \phi^{4}$ theory. The solid line represents the propagator $G(x, y)$. There are two kinds of vertices: a three-particle vertex proportional to $\lambda \phi$ and a four-particle vertex.

Therefore, the CJT effective potential at finite temperatures in the Hartree-Fock approximation is derived

$$
\begin{aligned}
V_{\beta}^{C J T}\left(\phi_{0}, G\right)= & \delta \Omega+\frac{m^{2}+\delta m^{2}}{2} \phi_{0}^{2}+\frac{\lambda+\delta \lambda}{24}\left(\phi_{0}^{2}\right)^{2} \\
& +\frac{1}{2} \int_{\beta} \operatorname{tr} \ln G^{-1}(k)+\frac{1}{2} \int_{\beta} \operatorname{tr}\left[D^{-1}\left(k ; \phi_{0}\right) G\right] \\
& +\frac{\lambda+\delta \lambda}{24}\left\{\left[\int_{\beta} G_{\alpha \alpha}(k)\right]^{2}+2 \int_{\beta} G_{\alpha \beta}(k) \int_{\beta} G_{\beta \alpha}(k)\right\} .
\end{aligned}
$$


Minimizing $V_{\beta}^{C J T}\left(\phi_{0}, G\right)$ with respect to $G_{\alpha \beta}(k)$ we obtain the Schwinger-Dyson (SD) equations for propagators

$$
G_{\alpha \beta}^{-1}(p)=D_{\alpha \beta}^{-1}\left(p ; \phi_{0}\right)+\frac{\lambda+\delta \lambda}{6}\left[\delta_{\alpha \beta} \int_{\beta} G_{\delta \delta}(k)+2 \int_{\beta} G_{\alpha \beta}(k)\right] .
$$

Basing on the structure of (II.6) and the $O(4)$ symmetry we adopt the following ansatz for propagators $G_{\alpha \beta}$ in Hartree-Fock approximation

$$
G_{\alpha \beta}^{-1}(p)=\left(p^{2}+X^{2}\right) \delta_{\alpha \beta}+Y^{2} \frac{\phi_{0}^{\alpha} \phi_{0}^{\beta}}{\phi_{0}^{2}} .
$$

Inserting (II.7) into (II.5) provides

$$
\begin{aligned}
V_{\beta}^{C J T}\left(\phi_{0}, M_{\sigma}, M_{\pi}\right) & =\delta \Omega+\frac{m^{2}+\delta m^{2}}{2} \phi_{0}^{2}+\frac{\lambda+\delta \lambda}{24}\left(\phi_{0}^{2}\right)^{2}+Q\left(M_{\sigma}\right)+3 Q\left(M_{\pi}\right) \\
& +\frac{1}{2}\left(m^{2}+\delta m^{2}+\frac{\lambda+\delta \lambda}{2} \phi_{0}^{2}-M_{\sigma}^{2}\right) P\left(M_{\sigma}\right) \\
& +\frac{3}{2}\left(m^{2}+\delta m^{2}+\frac{\lambda+\delta \lambda}{6} \phi_{0}^{2}-M_{\pi}^{2}\right) P\left(M_{\pi}\right) \\
& +\frac{\lambda+\delta \lambda}{24}\left\{3\left[P\left(M_{\sigma}\right)\right]^{2}+15\left[P\left(M_{\pi}\right)\right]^{2}+6 P\left(M_{\sigma}\right) P\left(M_{\pi}\right)\right\},
\end{aligned}
$$

where

$$
\begin{aligned}
& M_{\sigma}=X^{2}+Y^{2}, \\
& M_{\pi}=X^{2}
\end{aligned}
$$

and

$$
\begin{aligned}
& Q(M)=\frac{1}{2} \int_{\beta} \ln \left(k^{2}+M^{2}\right), \\
& P(M)=\int_{\beta} \frac{1}{k^{2}+M^{2}} .
\end{aligned}
$$

In order to regularize the divergent integrals $P(M)$ and $Q(M)$, contained in the expression (II.8) of the effective potential, let us use the three-dimensional momentum cutoff scheme, in which every divergent integral is written as the sum of a divergent part and a finite part, namely

$$
\begin{aligned}
Q(M) & =\operatorname{Div} Q(M)+Q_{f}(M), \\
\operatorname{Div} Q(M) & =-\frac{M^{4}}{4} I_{2}+\frac{M^{2}}{2} I_{1}, \\
Q_{f}(M) & =\frac{M^{4}}{64 \pi^{2}}\left(\ln \frac{M^{2}}{\mu^{2}}-\frac{1}{2}\right)+T \int \frac{d^{3} k}{(2 \pi)^{3}} \ln \left(1-e^{-\frac{E(\vec{k})}{T}}\right), \\
P(M) & =\operatorname{Div} P(M)+P_{f}(M), \\
\operatorname{Div} P(M) & =I_{1}-M^{2} I_{2}, \\
P_{f}(M) & =\frac{M^{2}}{16 \pi^{2}} \ln \frac{M^{2}}{\mu^{2}}-\int \frac{d^{3} k}{(2 \pi)^{3}}\left[E(\vec{k})\left(1-e^{\frac{E(\vec{k})}{T}}\right)\right]^{-1},
\end{aligned}
$$




$$
I_{1}=\frac{\Lambda^{2}}{8 \pi^{2}}, \quad I_{2}=\frac{1}{16 \pi^{2}} \ln \frac{\Lambda^{2}}{\mu^{2}}, \quad E(\vec{k})=\left(\vec{k}^{2}+M^{2}\right)^{1 / 2} .
$$

Now the renormalization is carried out so that all infinite terms appearing in (II.8) must be eliminated. For this end, the counterterms $\delta \Omega, \delta m^{2}$ and $\delta \lambda$ are chosen to obey the following constraints:

$$
\begin{aligned}
& \delta \Omega+\operatorname{Div} Q\left(M_{\sigma}\right)+3 \operatorname{Div} Q\left(M_{\pi}\right)=0, \\
& \frac{\delta m^{2}}{2}\left[\phi_{0}^{2}+P\left(M_{\sigma}\right)+3 P\left(M_{\pi}\right)\right] \\
& +\frac{\delta \lambda}{4}\left\{\frac{\left(\phi_{0}^{2}\right)^{2}}{6}+\phi_{0}^{2} P\left(M_{\sigma}\right)+\phi_{0}^{2} P\left(M_{\pi}\right)+\frac{1}{2}\left[P\left(M_{\sigma}\right)\right]^{2}+\frac{5}{2}\left[P\left(M_{\pi}\right)\right]^{2}+P\left(M_{\sigma}\right) P\left(M_{\pi}\right)\right\} \\
& +\frac{1}{2}\left(m^{2}+\frac{\lambda}{2} \phi_{0}^{2}-M_{\sigma}^{2}\right) \operatorname{Div} P\left(M_{\sigma}\right)+\frac{3}{2}\left(m^{2}+\frac{\lambda}{6} \phi_{0}^{2}-M_{\pi}^{2}\right) \operatorname{Div} P\left(M_{\pi}\right) \\
& +\frac{\lambda}{24}\left\{3 \operatorname{Div}\left[P\left(M_{\sigma}\right)\right]^{2}+15 \operatorname{Div}\left[P\left(M_{\pi}\right)\right]^{2}+6 \operatorname{Div}\left[P\left(M_{\sigma}\right) P\left(M_{\pi}\right)\right]\right\}=0
\end{aligned}
$$

(II.10) is a linear equation of two unknowns $\delta m^{2}$ and $\delta \lambda$, it has an infinite number of roots. In the following are two simplest cases:

a) $\delta m^{2}=0$

b) $\delta \lambda=0$.

In the first case $\delta \lambda$ tends to a finite limit as $\Lambda \rightarrow+\infty$ and in the second case $\delta m^{2}$ tends to an infinity as $\Lambda \rightarrow+\infty$. As a rule, both cases are equally accepted for renormalizing the CJT effective potential in Hartree-Fock approximation. In general, the counterterms are temperature dependent as was mentioned in [14].

We obtain ultimately the renormalized effective potential at finite temperatures

$$
\begin{aligned}
V_{\beta}^{C J T}\left(\phi_{0}, M_{\sigma}, M_{\pi}\right)= & \frac{m^{2}}{2} \phi_{0}^{2}+\frac{\lambda}{24}\left(\phi_{0}^{2}\right)^{2}+Q_{f}\left(M_{\sigma}\right)+3 Q_{f}\left(M_{\pi}\right) \\
& +\frac{1}{2}\left(m^{2}+\frac{\lambda}{2} \phi_{0}^{2}-M_{\sigma}^{2}\right) P_{f}\left(M_{\sigma}\right)+\frac{3}{2}\left(m^{2}+\frac{\lambda}{6} \phi_{0}^{2}-M_{\pi}^{2}\right) P_{f}\left(M_{\pi}\right) \\
& +\frac{\lambda}{8}\left\{\left[P_{f}\left(M_{\sigma}\right)\right]^{2}+5\left[P_{f}\left(M_{\pi}\right)\right]^{2}+2 P_{f}\left(M_{\sigma}\right) P_{f}\left(M_{\pi}\right)\right\} . \quad \text { (II.11) }
\end{aligned}
$$

The SD equations for $M_{\sigma}^{2}$ and $M_{\pi}^{2}$ and the gap equation for the sigma condensate are, respectively, derived from (II.11) for $\phi_{0}^{4}=\sigma, \phi_{0}^{i}=\pi^{i}=0$,

$$
\begin{aligned}
& M_{\sigma}^{2}=m^{2}+\frac{\lambda}{2} \sigma_{0}^{2}+\frac{\lambda}{2} P_{f}\left(M_{\sigma}\right)+\frac{\lambda}{2} P_{f}\left(M_{\pi}\right), \\
& M_{\pi}^{2}=m^{2}+\frac{\lambda}{6} \sigma_{0}^{2}+\frac{\lambda}{6} P_{f}\left(M_{\sigma}\right)+\frac{5 \lambda}{6} P_{f}\left(M_{\pi}\right),
\end{aligned}
$$

and

$$
\left(\frac{d V_{\beta}^{C J T}}{d \phi_{0}^{4}}\right)_{\substack{\phi_{0}^{4} \\ \phi_{0}^{i}=0}}=\sigma_{0}\left(m^{2}+\frac{\lambda}{6} \sigma_{0}^{2}+\frac{\lambda}{2} P_{f}\left(M_{\sigma}\right)+\frac{\lambda}{2} P\left(M_{\pi}\right)\right)=0,
$$

or 


$$
m^{2}+\frac{\lambda}{6} \sigma_{0}^{2}+\frac{\lambda}{2} P_{f}\left(M_{\sigma}\right)+\frac{\lambda}{2} P\left(M_{\pi}\right)=0
$$

Taking into account (II.12) the equation (II.14) reduces to

$$
\frac{\lambda}{3} \sigma_{0}^{2}-M_{\sigma}^{2}=0
$$

Adopting the argument of [19] we distinguish the effective masses of mesons, $M_{\sigma}$ and $M_{\pi}$, as those appearing in the approximate propagators, from the physical masses which are defined as the curvatures of the effective potential around its minimum,

$$
\begin{aligned}
& m_{\sigma}^{2} \stackrel{\text { def. }}{=}\left(\frac{d^{2} V_{\beta}^{C J T}}{d \sigma^{2}}\right) \begin{array}{l}
\sigma=\sigma_{0} \\
\pi=0
\end{array}, \\
& m_{\pi}^{2} \stackrel{\text { def. }}{=}\left(\frac{d^{2} V_{\beta}^{C J T}}{d \pi^{2}}\right)_{\begin{array}{l}
\sigma \\
\sigma=\sigma_{0} \\
\pi=0
\end{array}}=0,
\end{aligned}
$$

in the broken symmetry phase.

Equations (II.12)-(II.15) enable us to determine the "transition temperature" $T_{c 1}$, at which the broken chiral symmetry is restored: $\sigma_{0}, M_{\sigma}$ and $M_{\pi}$ tend to zero as $T \rightarrow T_{c 1}-0$. Indeed, Eq. (II.15) tells that $M_{\sigma} \rightarrow 0$ as $\sigma_{0} \rightarrow 0$ and Eq. (II.13) simplifies to

$$
M_{\pi}^{2}=m^{2}+\lambda P_{f}\left(M_{\pi}\right)
$$

When $M_{\pi}$ vanishes as $T \rightarrow T_{c 1}$, i.e. $M_{\pi} / T_{c 1} \ll 1$, we have the expression

$$
P_{f}\left(M_{\pi}\right) \approx T_{c 1}^{2}\left[\frac{1}{12}-\frac{1}{4 \pi} \frac{M_{\pi}}{T_{c 1}}-\frac{1}{16 \pi^{2}} \frac{M_{\pi}^{2}}{T_{c 1}^{2}} \ln \frac{M_{\pi}^{2}}{T_{c 1}^{2}}\right] .
$$

Combining (II.18) and (II.19) leads to

$$
T_{c 1} \approx \sqrt{2}\left(-\frac{6 m^{2}}{\lambda}\right)^{1 / 2}=\sqrt{2} f_{\pi} \approx 131.5 \mathrm{MeV} .
$$

Here $f_{\pi}=93 \mathrm{MeV}$ is the pion decay constant.

\section{NUMERICAL STUDY IN HARTREE-FOCK APPROXIMATION}

Let us first introduce a term explicitly breaking chiral symmetry into the lagrangian density

$$
\mathcal{L}=\frac{1}{2}\left(\partial_{\mu} \phi^{\alpha}\right)^{2}+\frac{m^{2}+\delta m^{2}}{2} \phi^{2}+\frac{\lambda+\delta \lambda}{24}\left(\phi^{2}\right)^{2}+\delta \Omega-c \sigma
$$

The renomalized effective potential corresponding to (III.1) reads 


$$
\begin{aligned}
V_{\beta}^{C J T}\left(\sigma_{0}, M_{\sigma}, M_{\pi}\right)= & \frac{m^{2}}{2} \sigma_{0}^{2}+\frac{\lambda}{24} \sigma_{0}^{4}-c \sigma_{0}+Q_{f}\left(M_{\sigma}\right)+3 Q_{f}\left(M_{\pi}\right) \\
& +\frac{1}{2}\left(m^{2}+\frac{\lambda}{2} \sigma_{0}^{2}-M_{\sigma}^{2}\right) P_{f}\left(M_{\sigma}\right)+\frac{3}{2}\left(m^{2}+\frac{\lambda}{6} \sigma_{0}^{2}-M_{\pi}^{2}\right) P_{f}\left(M_{\pi}\right) \\
& +\frac{\lambda}{8}\left\{\left[P_{f}\left(M_{\sigma}\right)\right]^{2}+5\left[P_{f}\left(M_{\pi}\right)\right]^{2}+2 P_{f}\left(M_{\sigma}\right) P_{f}\left(M_{\pi}\right)\right\},
\end{aligned}
$$

which leads to the gap equation for the sigma condensate $\sigma_{0}$

$$
\sigma_{0}\left(M_{\sigma}^{2}-\frac{\lambda}{3} \sigma_{0}^{2}\right)=c .
$$

The SD equations for $M_{\sigma}$ and $M_{\pi}$, derived from (III.2), have the same form (II.12) and (II.13). Inserting these equations into (III.2) we arrive at

$$
\begin{aligned}
V\left(\sigma_{0}, T\right)= & \frac{m^{2}}{2} \sigma_{0}^{2}+\frac{\lambda}{24} \sigma_{0}^{4}-c \sigma_{0}+Q_{f}\left(M_{\sigma}\right)+3 Q_{f}\left(M_{\pi}\right) \\
& -\frac{\lambda}{8}\left[P_{f}\left(M_{\sigma}\right)\right]^{2}-\frac{5 \lambda}{8}\left[P_{f}\left(M_{\pi}\right)\right]^{2}-\frac{\lambda}{4} P_{f}\left(M_{\sigma}\right) P_{f}\left(M_{\pi}\right) .
\end{aligned}
$$

Now the physical mass of pion no longer vanishes in broken symmetry phase and equals to

$$
m_{\pi}^{2}=\frac{c}{\sigma_{0}}
$$

For numerical computation we use the model parameters at zero temperature as initial condition, namely

$$
\begin{aligned}
& c=f_{\pi} m_{\pi}^{2} \quad \text { at } T=0 \\
& \lambda=\frac{3}{f_{\pi}^{2}}\left(m_{\sigma}^{2}-m_{\pi}^{2}\right) \quad \text { at } T=0 \\
& m^{2}=-\frac{1}{2} m_{\sigma}^{2}+\frac{3}{2} m_{\pi}^{2} \quad \text { at } T=0 .
\end{aligned}
$$

We take $m_{\pi}=138 \mathrm{MeV}, m_{\sigma}=500 \mathrm{MeV}$ and $f_{\pi}=93 \mathrm{MeV}$ at $T=0$.

For convenience, let us consider two separate cases.

\section{A. Hartree-Fock approximation in chiral limit $c=0$}

In addition to the preceding parameters, the regularization introduced another parameter $\mu^{2}$, which corresponds to renormalization scale. Therefore, we must first determine a suitable value $\mu_{0}^{2}$ for $\mu^{2}$, then all other quantities are determined at $\mu^{2}=\mu_{0}^{2}$. The value $\mu_{0}^{2}$ is determined as the real root of the following equation

$$
\left.\sigma_{0}\left(\mu^{2}, 0\right)\right|_{\mu^{2}=\mu_{0}^{2}}=f_{\pi}=93 \mathrm{MeV}
$$

where $\sigma_{0}\left(\mu^{2}, 0\right)$ is a solution of the system of equations (II.12), (II.13) and (II.14) at $T=0$. 
The numerical computation gives $\mu_{0}=539.6 \mathrm{MeV}^{1}$. Eliminating $\sigma_{0}$ from (II.12)(II.14) we get

$$
\begin{aligned}
& M_{\sigma}^{2}=-2 m^{2}-\lambda P_{f}\left(M_{\sigma}\right)-\lambda P_{f}\left(M_{\pi}\right), \\
& M_{\pi}^{2}=-\frac{\lambda}{3} P_{f}\left(M_{\sigma}\right)+\frac{\lambda}{3} P_{f}\left(M_{\pi}\right) .
\end{aligned}
$$

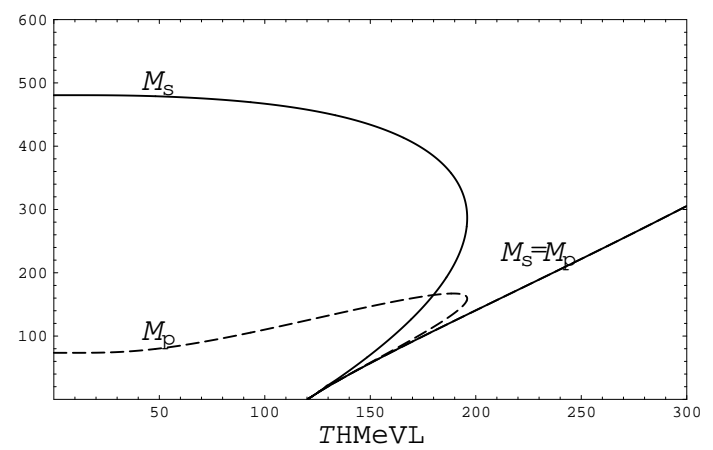

FIG. 2. The temperature dependent solution of the SD equations in the chiral limit.

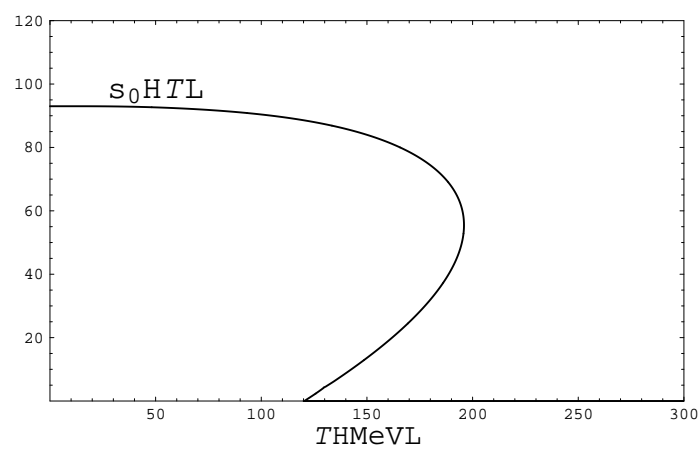

FIG. 3. Evolution of the order parameter as a function of temperature.

Inserting $\mu=\mu_{0}$ into the system (III.6) and then solving numerically the preceding system of two equations we obtain the solution presented in Fig. 2. As is shown in Fig. 3 the temperature $T_{c 1}$, calculated above by means of the high temperature approximation, is very close to the one, obtained by numerically solving the system (II.12)-(II.14). As the temperature increases from zero, the order parameter decreases from $f_{\pi}$, jumps to zero at $T_{c 1}$ and remains zero above $T_{c 1}$. In the meanwhile, the effective masses of mesons, $M_{\sigma}$ and $M_{\pi}$, change along the upper lines, jump to lower coinciding line at $T_{c 1}$ and increase along this line. It is clear that the phase transition is of first order. The indication of a first order phase transition has been reported in many publications [14-20].

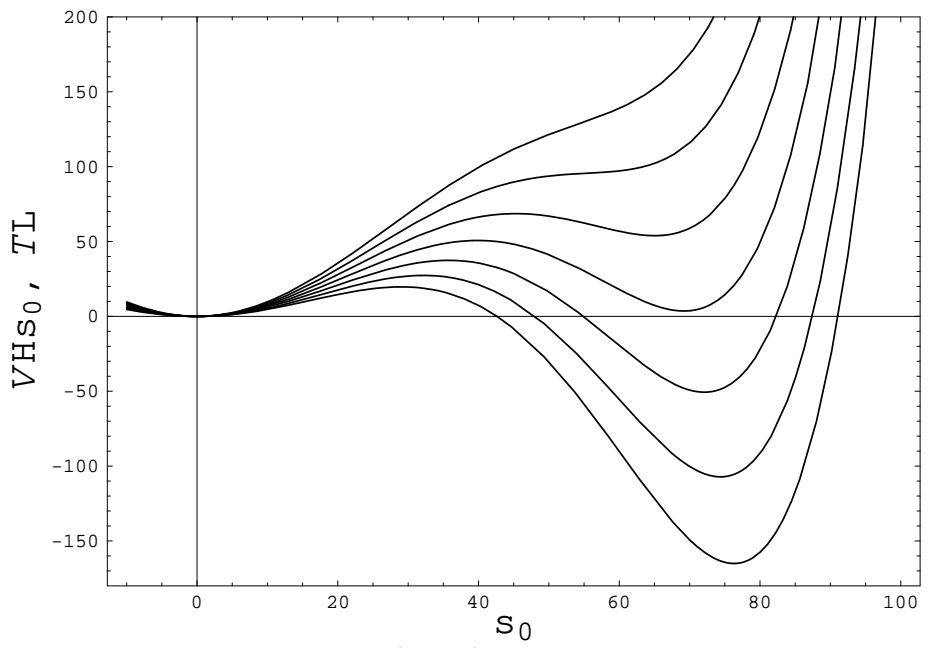

FIG. 4. Evolution of the effective potential $V\left(\sigma_{0}, T\right)$ as a function of the order parameter $\sigma_{0}$ for several temperature steps: $T=197,193,189,185,181,177,173 \mathrm{MeV}$ from top to bottom. Two minima of $V\left(\sigma_{0}, T\right.$ appear as degenerate at $T_{c} \approx 185 \mathrm{MeV}$.

\footnotetext{
${ }^{1}$ which corresponds to $M_{\sigma}=481.58 \mathrm{MeV}, M_{\pi}=73.53 \mathrm{MeV}$ at $T=0$.
} 
Next let us compute numerically the effective potential $V\left(\sigma_{0}, T\right)$, given by (III.4), as a function of the temperature and the order parameter. This will give more insight into the nature of phase transition. In Fig. 4 is depicted the evolution of $V\left(\sigma_{0}, T\right)$ against $\sigma_{0}$ for several temperature steps. It is found that the two minima of $V\left(\sigma_{0}, T\right)$ appear as degenerate at $T_{c 2} \approx 185 \mathrm{MeV}$. For $T \gtrsim 193 \mathrm{MeV}, V\left(\sigma_{0}, T\right)$ has only one minimum at $\sigma_{0}=0$. The shape of the potential confirms that a first order phase transition occurs.

\section{B. Hartree-Fock approximation in the broken symmetry case $c \neq 0$}

For completeness let us now consider the case $c \neq 0$, hence the gap equation for the order parameter (II.14) turns out to be

$$
\left[m^{2}+\frac{\lambda}{6} \sigma_{0}^{2}+\frac{\lambda}{2} P_{f}\left(M_{\sigma}\right)+\frac{\lambda}{2} P_{f}\left(M_{\pi}\right)\right] \sigma_{0}-c=0,
$$

and the SD equations (II.12), (II.13) remain unchanged.

After solving numerically the system (II.12), (II.13) and (III.7) we get the solution presented in Fig. 5 .
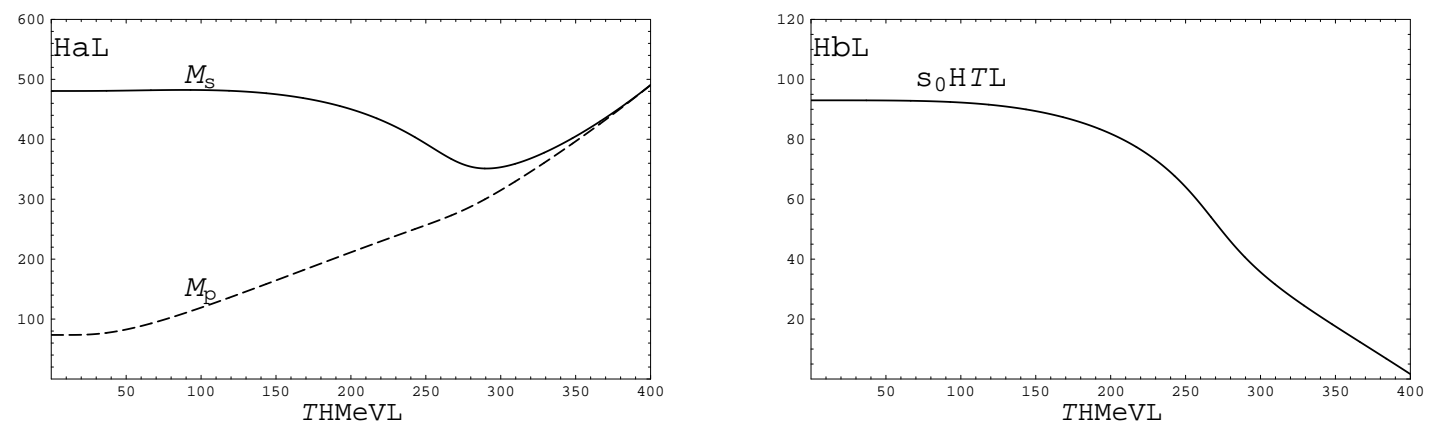

FIG. 5. (a) Solution of the system of gap equations for $c \neq 0$. At low temperature $M_{\pi}$ coincides with the observed $m_{\pi}$. (b) Evolution of the order parameter as a function of temperature.

Comparing the above presented result with those obtained in [16-19] we have an excellent agreement which shows that the quantum effect is an negligible quantity.

\section{THE HIGHER-LOOP DIAGRAMS CONTRIBUTION}

In the preceding section it was shown that the phase transition is of first order. The Gaussian approximation [24] in 3+1 dimensions gives the same result. However, the renormalization group approach applied to the linear sigma model [25, 26] indicates that the phase transition is of second order. Arnold and Espinosa [27] pointed out that loop diagrams other than superdaisy ones are important near critical temperature. The nature of the phase transition in the $\phi^{4}$ field theory remains a basic question to be settled [28]. Therefore, this requires a further investigation on higher-loop effect. In this respect, it is necessary to incorporate the higher-loop diagrams into consideration. The higher-loop graph next to the double bubble one is the sunset graph given in Fig 6 


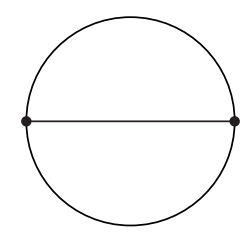

FIG. 6. The sunset graph.

In a recent publication [29] Bordag and Skalozub studied, in addition to the double bubble, an infinite series of graphs shown in Fig 7

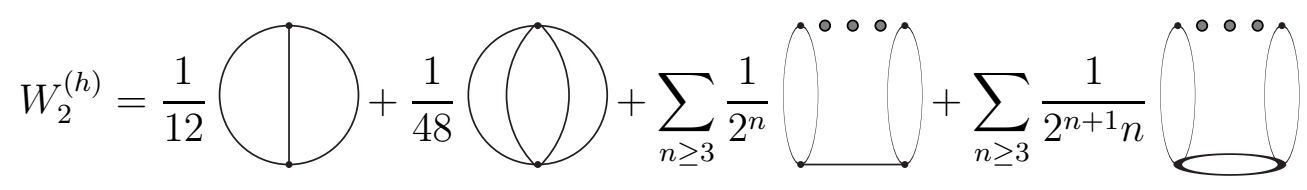

FIG. 7. The big dots symbolize further insertion of the subgraph $-\bigcirc-$ so that the number of vertices is $n$. The 3-particle vertex $\sim-\lambda \phi_{0}$, the 4 -particle vertex $\sim-\lambda$.

which is motivated by the $1 / N$ expansion.

Making use of the ansatz

$$
G^{-1}(p)=p^{2}+M^{2}
$$

at high temperature they concluded that the contribution of this set of graphs does not influence significantly on the physical process, making the transition a bit stronger first order.

Next let us generalize the consideration including an infinite series of diagrams depicted in Fig 8

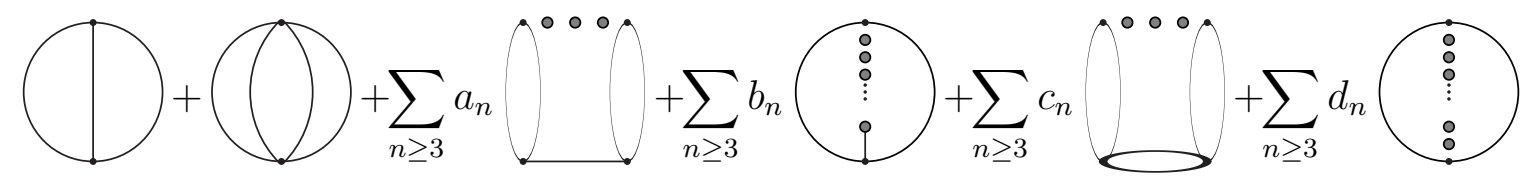

FIG. 8. The big dots symbolize further insertion of the subgraph $-\bigcirc-$ so that the number of vertices is $n$.

Then the effective potential, corresponding to Fig 8, reads

$$
V_{2}^{[8]}=\frac{3}{2} \lambda^{2} \phi_{0}^{2} H+\frac{\lambda^{2}}{2} K
$$

where

$$
\begin{aligned}
H & =\operatorname{Tr}_{q} G(q) \Sigma(q)\left\{f_{1}[\lambda \Sigma(q)]+f_{2}[\lambda \Sigma(q)]\right\} \\
K & =\operatorname{Tr}_{q} \Sigma^{2}(q)\left\{g_{1}[\lambda \Sigma(q)]+g_{2}[\lambda \Sigma(q)]\right\} \\
\Sigma(p) & =\operatorname{Tr}_{q} G(q) G(p+q)=-\bigcirc-
\end{aligned}
$$

and $f_{1}, f_{2}, g_{1}, g_{2}$ are respectively the functions representing the series expansions given in Fig 8 with the coefficients $a_{n}, b_{n}, c_{n}, d_{n}$. 
It is evident that

$$
f_{i}[0]=g_{i}[0]=1, \quad i=1,2 .
$$

Now it is easily seen that we arrive at the same conclusion by applying the discussion of [29] to every fixed expression for every function among $f_{1}, f_{2}, g_{1}$ and $g_{2}$.

\section{v. CONCLUSION AND DISCUSSION}

We have considered the $O(4)$ linear sigma model at finite temperature within the framework of the CJT formalism restricted to the double bubble diagram approximation. The renormalization of the CJT effective potential for this model has been discussed in many papers [14-19] and one succeeds only in the large- $N$ limit case [23], where all divergent terms are absorbed into the bare quantities. For other case, however, not all divergences can be removed away [15]. In the present paper, following exactly the spirit of renormalization that requires all divergent terms must be absorbed into counterterms, corresponding to renormalizing mass and coupling constant, we impose two constraints (II.9) and (II.10), to ensure that only finite terms would be still present in the effective potential. It is possible that this renormalization method is most suited for renormalizing the CJT effective potential in loop approximations. In order for the Goldstone theorem not to be violated the physical masses of mesons are defined by (II.16), (II.17) and propagators are chosen in the symmetric form (II.7). The solution of the gap equations, derived from the renormalized effective potential, and the shape of the effective potential, as a function of the order parameter exhibits a first order phase transition with the critical temperature $T_{c} \simeq 185 \mathrm{MeV}$.

To go further to higher-loop graphs we have shown that the order of the phase transition is unchanged by incorporating an infinite series of diagrams, in which the sunset one is a special case.

In summary, it is possible to confirm that the phase transition in the $\phi^{4}$ field theory is of first order.

\section{ACKNOWLEDGMENTS}

The financial support from the Vietnam National Science Foundation is acknowledged.

[1] D. E. Brahm and S. D. H. Hsu, Report No. CALT-68-1705/HUTP-91-A063 (1991) (unpublished).

[2] See for instance, J. Wambach, in Proc. of Quark Matter 97, Nucl. Phys. A638 (1991) 171c.

[3] See for instance, K. Rajagopal, in Quark-Gluon Plasma 2, edited by R. Hwa (World Scientific, Singapore, 1995) 484.

[4] See for instance, Proc. of Lattice 96, Nucl. Phys. B53 (Proc. Suppl.) (1997) 1.

[5] Quark Matter '99, Nucl. Phys. A661 (1999).

[6] D. A. Kizhnits and A. D. Linde, Phys. Lett. B42 (1972) 471.

[7] S. Weinberg, Phys. Rev. D9 (1974) 3357.

[8] L. Doland and R. Jackiw, Phys. Rev. D9 (1974) 3320.

[9] J. M. Cornwall, R. Jackiw and E. Tomboulis, Phys. Rev. D15 (1974) 2428.

[10] G. Amelino-Camelia and So-Young Pi, Phys. Rev. D47 (1992) 2356.

[11] P. Castorina, M. Consoli and D. Zappala, Phys. Lett. B201 (1988) 90. 
[12] C. G. Boyd, D. E. Brahm and S. D. Hsu, Phys. Rev. D48 (1993) 4963.

[13] M. Quiros, hep-ph/9304284.

[14] G. Baym and G. Grinstein, Phys. Rev. D15 (1977) 2897.

[15] G. Amelino-Camelia, Phys.Lett. B407 (1997) 268 (hep-ph/9702403).

[16] J. T. Lenaghan and D. H. Rischke, J.Phys. G26 (2000) 431 (nucl-th/9901049).

[17] Heni-Seol Roh and T. Matsui, Eur.Phys.J. A1 (1998) 205 (nucl-th/9611050).

[18] N. Petropoulos, J.Phys. G25 (1999) 2225 (hep-ph/9807331).

[19] Y. Nemoto, K. Naito and M. Oka, Eur. Phys. J. A9 (2000) 245.

[20] N. Bilic and H. Nicolic, Eur. Phys. J. C6 (1999) 515.

[21] O. Eboli, R. Jackiw and S-Y. Pi, Phys. Rev. D37 (1988) 3357.

[22] See for instance, J. I. Kapusta, Finite-Temperature Field Theory, Cambridge University Press (1989).

[23] S. Coleman, R. Jackiw and H. D. Politzer, Phys. Rev. D10 (1974) 2491.

[24] P. M. Stevenson, Phys. Rev. D32 (1985) 1389, and references therein.

[25] K. Rajagopal and F. Wilczek, Nucl. Phys. B399 (1993) 395.

T. Umekawa, K. Naito and M. Oka, hep-ph/9905502.

[26] K. Ogure and S. Sato, Phys. Rev. D58 (1998) 85010.

[27] P. Arnold and O. Espinosa, Phys. Rev. D47 (1993) 3546.

[28] M. Consolo and P. M. Stevenson, Int.J.Mod.Phys. A15 (2000) 133 (hep-ph/9905427).

[29] M. Bordag and V. Skalozub, J.Phys. A34 (2001) 461 (hep-th/0006089). 